\title{
Measuring Oxytocin: The Blind Men and the Elephant?
}

Evan L MacLean ${ }^{1 *}$, Steven Wilson ${ }^{2}$, W. Lance Martin ${ }^{3}$

John M. Davis ${ }^{4}$, Hossein P. Nazarloo ${ }^{5}$ \& C. Sue Carter ${ }^{5}$

${ }^{1}$ School of Anthropology \& Department of Psychology, University of Arizona

2 Department of Chemistry, University of Oslo

${ }^{3}$ Martin Protean, LLC

${ }^{4}$ Psychiatric Institute, University of Illinois at Chicago

${ }^{5}$ The Kinsey Institute, Indiana University

\section{* Address for correspondence}

Evan MacLean

evanmaclean@email.arizona.edu

520-621-0386

University of Arizona

P.O. Box 210030

Tucson, AZ 85721-0030 


\begin{abstract}
Since its discovery more than a century ago, oxytocin has become one of the most intensively studied molecules in behavioral biology. In the last five years, Psychoneuroendocrinology has published more than 500 articles with oxytocin in the title, with many of these articles including measures of endogenous oxytocin concentrations. Despite longstanding interest, methods of measuring endogenous oxytocin are still in active development. The widely varying oxytocin concentrations detected by different approaches to measurement - and lack of correlation among these techniques - has led to controversy and confusion. We identify features of oxytocin that may help to explain why various approaches may be differentially sensitive to diverse conformational states of the oxytocin molecule. We propose that discrepancies in data generated by different methods of measurement are not necessarily an indicator that some methods are valid whereas others are not. Rather, we propose that current challenges in the measurement of oxytocin may be analogous to the parable of the blind men and the elephant, with different methods of sample preparation and measurement being sensitive to different states in which the oxytocin molecule can exist.
\end{abstract}


Since its discovery more than a century ago, oxytocin has become one of the most intensively studied molecules in behavioral biology. Once thought of primarily as a female reproductive hormone, oxytocin is now recognized for having diverse roles in the mammalian nervous system, and is implicated in processes ranging from social monogamy and theory of mind to regulation of appetite, the immune and autonomic systems, and bone mass and cardiomyocyte differentiation (Blackburn, Demko, Hoffman, Stricker, \& Verbalis, 1992; Carter, 2014; Carter \& Perkeybile, 2018; Feldman, Weller, Zagoory-Sharon, \& Levine, 2007; Li, Wang, Wang, \& Wang, 2017; MacKinnon et al., 2018; Paquin, Danalache, Jankowski, McCann, \& Gutkowska, 2002; Peltola, Strathearn, \& Puura, 2018; Tamma et al., 2009; Wai, Siu, Zhang, \& Chan, 2018). In the last five years, Psychoneuroendocrinology has published more than 500 articles with oxytocin in the title, with many of these articles including measures of endogenous oxytocin concentrations. Despite this longstanding interest, methods of measuring endogenous oxytocin are still in active development, and questions about the validity of various techniques have spurred controversy and confusion (Carter et al., 2007; Carter, 2014; Jurek \& Neumann, 2018; Leng \& Sabatier, 2016; McCullough, Churchland, \& Mendez, 2013).

Specifically, two of the most common approaches for sample preparation (use of extracted or non-extracted samples; see below for details) lead to entirely uncorrelated results (Leng \& Sabatier, 2016); even when using the same protocols, different commercially available assays can produce markedly divergent measurements (Lefevre et al., 2017; MacLean et al., 2018). Questions about endogenous oxytocin concentrations have been further complicated by recent findings that the vast majority of endogenous oxytocin may be bound to other molecules in plasma and serum. In many cases oxytocin may evade detection unless these bonds are broken prior to measurement (Brandtzaeg et al., 2016; Martin \& Carter, 2013). Although efforts to develop reliable measures / confirm the validity of bound oxytocin have proven challenging (Franke, Li, Menden, Lee, \& Lai, 2019), multiple techniques have been developed in recent years, with utility for both immunoassay and mass spectrometry applications (Brandtzaeg et al., 2016; Liu et al., 
2019). The widely varying oxytocin concentrations detected by different approaches to sample preparation and measurement - and lack of correlation between these techniques - has led to the suggestion that particular approaches may be 'no more than a random number generator' (Leng \& Sabatier, 2016). Reflecting on the poor agreement between various approaches, others have suggested that it is urgent that we adopt a single approach to be implemented as the standard in the field (McCullough et al., 2013). Although we agree that progress will depend on a better understanding of why different methods produce such different results, we suggest that it is premature to accept any single approach as a gold standard, since discrepancies between methods are not necessarily an indicator that some methods are valid whereas others are not. Instead, we suggest that the current challenges in the measurement of oxytocin may be analogous to the parable of the blind men and the elephant.

In this parable, a group of blind men conceptualize what an elephant is like by touching its body. Each man touches a different part of the elephant, and afterward recounts his perspective. Of course, all of the men find themselves in utter disagreement about what an elephant is like, having been exposed to only partial information about a complex system. We propose that the current diversity of approaches to measuring oxytocin may be analogous to the blind men in this parable. Varying approaches to sample preparation and measurement of oxytocin may yield different and often conflicting information due to differential sensitivity to diverse conformational states of the oxytocin molecule. We propose that this phenomenon arises because oxytocin, like an elephant, is biologically complex.

\section{Characteristics of the Oxytocin Molecule}

Oxytocin measured in plasma is reported to have a short half-life, ranging from $\sim 1-5$ minutes, and in vivo, is rapidly degraded by diverse peptidases in the passage of blood through the liver and kidneys (Chard, Boyd, Forsling, McNeilly, \& Landon, 1970; Morin et al., 2008; Rydén \& Sjöholm, 1969). But perhaps more importantly, oxytocin also strongly binds to other molecules (including itself), which are common in biological matrices (Avanti et al., 2013; Avanti et al., 2012; 
Brandtzaeg et al., 2016; Liu et al., 2019; Yamamoto et al., 2019). Although we know little about the physiological significance of bound oxytocin, recent work reveals that bound oxytocin can be transported across the blood-brain barrier yielding important consequences in the central nervous system. Even following sample collection, oxytocin binds slowly and non-specifically to plasma proteins, and as described below, a substantial amount of bound oxytocin can be discarded by common approaches to sample purification (Brandtzaeg et al., 2016; MacLean, Gesquiere, Gee, et al., 2017; Martin \& Carter, 2013). Collectively, these properties of oxytocin have major implications for issues related to its measurement.

\section{Measuring Oxytocin}

The most common approaches to measuring oxytocin involve immunoassay or mass spectrometry. These techniques detect analytes in fundamentally different ways, and as a result, are characterized by different strengths and limitations (Table 1). Immunoassays rely on binding between oxytocin and an antibody (Figure 1). Oxytocin antibodies vary in the epitopes they recognize, which can lead to variable results between immunoassays (Lefevre et al., 2017). Additionally, because detection is based on antibody binding, there is potential for a wide range of interferences (Tate \& Ward, 2004) ranging from cross reactivity (in which structurally similar analytes produce false positive signal via reaction with the antibody) to heterophilic interference (in which interfering substances prevent antibody binding, again generating false positive signal). Thus, the identity of what is measured using immunoassay is never determined with certainty (Park \& Kricka, 2013). Despite these limitations, immunoassays provide the advantage that oxytocin may be recognized when it is bound to other components of the matrix, present in multiple forms, or when the molecule is partially degraded. Thus, a lack of specificity can sometimes be advantageous, as evidenced through studies of hormones in which the least specific antibodies sometimes yield the strongest associations with related biological phenomena (Galeandro et al., 2014). 
Mass spectrometry (MS) is sometimes described as being a gold standard for the detection and quantitation of specific molecules. Indeed, relative to immunoassays, MS can be highly specific because it "weighs" molecules, providing information about the molecule's structure (Figure 2). However, there are also many potential pitfalls with MS. For instance, analytes of interest may be suppressed by other compounds (e.g. phospholipids, polymers from plastic tubes, or anticoagulants; Mei et al., 2003). Additionally, if an oxytocin molecule is bound to other components of the matrix (even another oxytocin molecule), the species may not be detectable. Finally, there are scenarios in which common mass spectrometers with low resolution can mix up compounds that have similar properties (e.g. near identical molecular weights). Thus, there are limitations unique to this platform, and oxytocin's affinity for binding to other molecules imposes notable challenges for measurement via MS.

\section{Sample preparation}

Beyond the specific detection platform, pre-analytical steps have the potential to dramatically influence what states of oxytocin are available for detection. Perhaps the most influential preanalytical step is sample extraction. In short, extraction refers to a range of approaches designed to eliminate interfering substances from a complex matrix, while retaining, and often concentrating the target analyte. The simplest oxytocin extractions involve protein precipitation, in which a solvent is used to precipitate serum/plasma/urine proteins from the matrix, which are then discarded, leaving only soluble molecules in the supernatant fraction. Although free oxytocin is highly soluble, oxytocin bound to proteins will co-precipitate, effectively being discarded. Given that the majority of oxytocin is bound in serum and plasma, this approach will retain only a small fraction of the total oxytocin content (Brandtzaeg et al., 2016). In addition, while protein precipitations are simple and cost effective, they can be subject to high variability and poor analyte recovery (Alshammari, Al-Hassan, Hadda, \& Aljofan, 2015; Hennion, 1999).

A more common preanalytical step involves solid-phase extraction (SPE). In SPE, a sample is passed through a cartridge containing a chromatographic sorbent which selectively 
retains molecules, often as a function of their polarity and affinity for lipids (Figure 3). The sorbent is then washed to remove potentially interfering substances, and finally, molecules retained by the sorbent are eluted using a solvent. Compared to liquid-liquid extractions, solid-phase extraction can be highly selective. However, the effectiveness of SPE depends critically on the chemistry between components of sample and the sorbent, and in the case of oxytocin, SPE procedures are typically designed with the properties of the free nine amino acid peptide in mind. However, as noted above, oxytocin interacts dynamically with other elements of the matrix, which can affect retention on the sorbent, or whether bound analytes are effectively eluted. Sample preparation protocols which are optimized for the "catch and release" of free oxytocin in a simple matrix may still eliminate large amounts of oxytocin which is present in other naturally occurring conformational states, effectively "throwing out both the baby and the bathwater".

In addition, SPE can also be subject to breakthrough effects, a scenario in which the target analyte is not effectively retained in the sorbent, or poor recovery if only a fraction of the analyte is successfully eluted (Figure 3). Regarding the latter pitfall, it has been noted that common protocols recommended by immunoassay kit manufacturers often yield poor recovery, and may require substantial methodological development and optimization by the end-user to achieve acceptable recovery (Bienboire-Frosini, Chabaud, Cozzi, Codecasa, \& Pageat, 2017). Indeed, different chromatographic sorbents designed to capture molecules via the same mechanism (e.g. polarity) often behave differently, each requiring optimization of various elements, including wash steps, flow rate, and solvent selection. Although there are several commonly recommended generic protocols for oxytocin extraction (often specified in immunoassay kit manuals), end users should not blindly adopt these techniques or assume they will yield high levels of performance across all applications.

For immunoassay applications, the eluant from SPE is typically evaporated to dryness, and later reconstituted in an assay buffer. To expedite this procedure, researchers often heat samples, creating conditions that can potentially degrade the oxytocin molecule. While steroid 
hormones are generally robust to these conditions (Ziegler \& Wittwer, 2005), the effect of heat and oxidation presents greater risk when working with peptides, which are readily degraded by these procedures. Lastly, evaporated samples may form a compact residue that is not easily resuspended, creating the potential for additional sample loss due to ineffective reconstitution. Thus, while extraction is often portrayed as a simple and routine clean-up procedure, it presents a range of potential pitfalls in the context of dynamic peptides such as oxytocin.

\section{Implications}

The issues outlined above reveal a multitude of factors which can influence oxytocin measurement, beginning at the preanalytical stages, and extending through the process of quantitation. Preanalytical steps may discard some of the most abundant forms of oxytocin, fail to satisfactorily recover the analyte, or degrade the molecule through heat and/or oxidation. Quantitation by immunoassay will depend on whether particular epitopes remain available for binding, which may depend on whether oxytocin engages in complexes with other elements of the matrix, which likely occur in diverse forms (e.g., dimers or trimers of the oxytocin molecule, or binding to various proteins or lipids in plasma, serum, urine, and perhaps even saliva).

Based on these complexities, we suggest that is not at present useful to make inferences about whether a particular measurement approach is valid based on the magnitude of concentrations it detects, or whether these values correspond with those from historical practices. In the elephant metaphor, this would be akin to anchoring all judgements to the perspective of a single blind man, who happened to be the first to touch the elephant. From this viewpoint, it becomes clear that current debates - especially those that are not informed by the complex chemistry of this molecule - about the 'correct' way to measure oxytocin, may be fundamentally misguided. We suggest that true progress will depend on better answers to questions about the states in which we intend to detect oxytocin when measuring it, the biological causes and consequences of these states, and how and why various approaches to measurement may be differentially sensitive to the above. 
One important implication of this perspective is that progress will depend on a clearer formulation of precisely what researchers intend to measure when they measure oxytocin. Discrepancies between the results of different methods are often interpreted as an indication that one measure must be erroneous. That is, differences between measures are attributed to error, because both measures should detect the same entity, yet one or both fails to do so accurately. We agree that if two measures are designed to detect the same entity, and measurements are discordant, this reflects a serious methodological problem. However, we suggest that a neglected possibility is that differences between measures may also reflect variation in the states in which oxytocin is detected. For example, if oxytocin is bound to other elements in a biological matrix, it may remain detectable following some approaches to sample preparation and measurement, but not others. If one measure detects free oxytocin, but another detects free and bound oxytocin, and these concentrations are not in agreement (or correlation), is one measure valid whereas the other is not? If so, which? Clearly the answer to this question depends on the taxonomy of states in which we intend to measure oxytocin, and at present this taxonomy is often poorly specified (but see Brandtzaeg et al., 2016; Green et al., 2001; MacLean, Gesquiere, Gruen, et al., 2017). If - as current data suggest - oxytocin is present in multiple dynamic states, and more than one of these states is biologically meaningful or with different receptor affinity (Jurek \& Neumann, 2018) it is possible that there will be multiple valid measures, but these measures will not covary. At present, variation in reported values has been attributed exclusively to measurement error, without considering the possibility that different approaches may be fundamentally attuned to different states of the same molecule. These may reflect adaptive or functional changes in the availability of oxytocin, which will be important to interpreting outcomes. Of course, our perspective does not imply that all existing measures are valid, nor that we should accept all findings at face value without skepticism, assuming that they all index some meaningful component of oxytocin biology. Indeed, as detailed below, the possibility of multiple valid measures underscores a need to critically validate the full range of techniques being employed. 
One concern arising from our perspective is that although it provides an explanation for why multiple valid measures may vary, it also increases the number of conceptual entities to be accounted for, each perhaps requiring its own physiological explanation. Although we acknowledge the large number combinatorial possibilities that could arise, we hypothesize that much of the currently reported variance is attributable to differences between detecting only the free vs. the free and bound fractions (see below for discussion of the potential significance of these fractions). Considering oxytocin's interactions with a biological matrix also raises important questions about how these dynamics may vary between matrices. Although researchers are often interested in oxytocin's effects in the central nervous system (CNS), in most studies it is not possible to directly measure oxytocin release in the brain or cerebrospinal fluid, and instead samples are collected from peripheral fluids including blood, urine, and saliva. Current studies have produced mixed results regarding the correlation between oxytocin concentrations in the CNS and these peripheral fluids (Lefevre et al., 2017; Valstad et al., 2017), consistent with data suggesting that central and peripheral release can occur through both independent and coordinated mechanisms (Jurek \& Neumann, 2018; Landgraf \& Neumann, 2004; Wotjak et al., 1998).

When considering peripheral matrices, researchers often assume that measures from blood are most reliable given oxytocin's direct release into the bloodstream from the neurohypophysis. However, from the perspective of matrix interactions, blood proteins readily sequester oxytocin leading to challenges for detection (Brandtzaeg et al., 2016; Martin \& Carter, 2013). Similarly plasma proteins may cause interference in immunoassays (Selby, 1999), and common procedures to remove these interferences may discard oxytocin, as reviewed above. More recently, researchers have begun to measure oxytocin in saliva, an approach that was originally met with great skepticism (Horvat-Gordon, Granger, Schwartz, Nelson, \& Kivlighan, 2005). One of the primary limitations of this approach is that oxytocin's mode of entry into saliva remains poorly understood. Nonetheless studies using mass spectrometry confirm the identity of 
oxytocin in saliva, and salivary oxytocin is responsive to stimuli known to induce oxytocin release, including lactation, exercise, stress, and sexual stimulation (Carter et al., 2007; de Jong et al., 2015; MacLean et al., 2018; White-Traut et al., 2009). In contrast to blood, saliva presents a relatively clean matrix, limiting opportunities for binding to large proteins, and permitting measurement without extraction (however, at present the pharmacokinetics of oxytocin in saliva remain poorly understood). Regarding the latter point, in contrast to blood, oxytocin concentrations measured in saliva are highly correlated regardless of whether or not a solid-phase extraction is employed (MacLean et al., 2018). Therefore, there are several considerations regarding peripheral fluids for measurement of oxytocin, including: i) correlation with concentrations in the CNS, ii) biological clearance / temporal resolution, iii) potential for binding to other components of the matrix, and iv) the presence of potential interferants during measurement.

Importantly, our perspective is not intended to provide a free pass on methodological rigor. While recognizing that there are perfectly reasonable explanations why multiple valid methods may produce divergent results, it remains vitally important that new methods are developed and validated in a rigorous way, with attention to both analytical and biological validity. From an analytical perspective, methods must be accurate, reproducible, and allow detection within a biologically meaningful range of concentrations. For immunoassays, analytical validation requires (at minimum) tests of: i) parallelism, typically tested by serial dilution of samples to ensure parallel displacement against the standard curve, ii) accuracy/recovery, in which samples are spiked with standards, to confirm a lack of antigen-antibody interference from the sample matrix, and iii) intraand inter-assay variation, in which the same samples are tested multiple times within and across assays to assess reproducibility (Andreasson et al., 2015). It should also be mentioned that these individual tests can also have limitations, e.g. the difficulties of obtaining an analyte-free matrix for testing accuracy and recovery (which is far simpler when designing an assay for a drug known to not be present in a "blank" sample). For mass spectrometry, analytical validation will also 
require addition and assessments of internal standards, and control over potential suppressants in the matrix (e.g. phospholipids).

Biological validation confirms that a measure is sensitive to relevant physiological processes. In the case of oxytocin, appropriate physiological events may include parturition, lactation, sexual stimulation, exercise, non-noxious physical contact, or psychosocial stress (Dawood, Raghavan, Pociask, \& Fuchs, 1978; de Jong et al., 2015; MacLean et al., 2018; MacLean, Gesquiere, Gee, et al., 2017; Matthiesen, Ransjö-Arvidson, Nissen, \& Uvnäs-Moberg, 2001; Stock \& Uvnäs-Moberg, 1988; White-Traut et al., 2009). However, as biomarkers, the significance of different measures of oxytocin remains poorly understood. We hypothesize that measures of free oxytocin may be most meaningful with respect to acute changes in response to specific stimuli. However, free oxytocin concentrations may also be affected by age, morbidity, or pharmacological compounds that displace oxytocin from proteins, introducing confounds with potentially large effects (Rowland, Tozer, Derendorf, \& Hochhaus, 2011). In contrast, we expect that measures incorporating bound oxytocin may provide a more robust measure of basal individual differences, and peptidergic activity across longer time horizons (Brandtzaeg et al., 2016; MacLean, Gesquiere, Gruen, et al., 2017). However, distinguishing whether different measures of oxytocin have different biological significance is one of the most important challenges presented by our perspective. At present we know little about the bioactivity of bound oxytocin. However, emerging work suggests that bound OT may have important functions, echoing findings from renewed interest in the properties of bound steroids (Caldwell \& Jirikowski, 2014; Caldwell et al., 2006). For example, Yamamoto et al. (2019) have recently shown that that oxytocin can cross the blood brain barrier by binding to receptor for advanced glycation end-products (RAGE), maintaining bioactivity in the central nervous system following this transport. Regardless of the bioactivity of bound oxytocin, free oxytocin can become bound relatively quickly following sample collection (Brandtzaeg et al., 2016). Therefore, while researchers may be most interested in the fraction of oxytocin that was free at the time of sample collection, much of this fraction may no 
longer be free at the time of quantitation. Consequently, we face important questions about the significance of different fractions of oxytocin not in only terms of their bioactivity, but also with respect to whether measured values accurately reflect an organism's physiological state at the time of sample collection. In many cases the goal of measurement is to provide insights into the relationship between a chemical factor (in this case oxytocin) and dynamic biological processes, such as behavioral or physiological processes that may be regulated by the molecule being studied. Thus, in this example, changes in a given process may be sensitive to different aspects of the availability of the molecule. For example, oxytocin binding to a receptor, may be relatively strong, while disulfide bonding to molecules in blood may be a more dynamic process affected by variables such as availability of oxygen, temperature and $\mathrm{pH}$. Blood-bound oxytocin could also serve as a reservoir of the peptide available as an anti-inflammatory agent (Szeto et al., 2013).

We urge readers to treat validation work addressing the criteria above as an essential component of any study measuring oxytocin. These studies should be conducted independently in each laboratory performing measurement work, and replicated for each unique species and biological matrix being studied. Importantly, it is not sufficient to cite data from other laboratories or manufacturers of consumable products used in oxytocin research as a shortcut around this process. In addition to ensuring data quality, the publication of validation and methodological studies will make the strengths and weaknesses of various approaches more transparent to readers, and draw attention to procedural details that could change outcomes. One possible solution for ensuring rigor, both in methods and their reporting, would be for journals to require that validation data and detailed methodological descriptions be included for studies involving measurement (e.g. following FDA or Eurachem guidelines regarding analytical validation).

At present, few methodological studies have addressed the chemistry of oxytocin, and have instead focused on whether methods produce concentrations that are consistent with historical data, or the extent to which methods create or mitigate interference during quantitation. 
We agree that interference is an important concern, but also note that simply because a method minimizes interference, does not imply that this method affords a biologically meaningful measure. Du Vigneaud's pioneering work, which lead to the synthesis of oxytocin, emerged by "following the sulfur trail" (du Vigneaud, 1956), and recent data indicate that oxytocin's disulfide bridge readily engages in complexes which can strongly influence measurement (Brandtzaeg et al., 2016; Martin \& Carter, 2013). Renewed focus on the chemistry of oxytocin will enable us to better understand this molecule's biological fate, and to develop methodological approaches for the detection of oxytocin under a wider range of biological conditions.

Lastly, although beyond the scope of the present paper, to fully understand the significance of peripheral measures of oxytocin it is important to recognize that oxytocin is a component of a phylogenetically ancient system that also includes vasopressin (Carter, 2017). Thus, both peptides, and the receptors for these molecules, will need to be considered as we try to understand the functions of this system (for excellent reviews see Grinevich, KnoblochBollmann, Eliava, Busnelli, \& Chini, 2016; Jurek \& Neumann, 2018)

In sum, we believe that the measurement of oxytocin presents complex challenges that we are only beginning to understand. Before assuming that discrepancies reflect errors or invalid measurements, we should consider the possibility that different measures capture different components of the biological story that oxytocin has to tell. In this sense, we are like the blind men in the parable. Rather than debating which blind man has the best perspective, we suggest that it will be most productive to pursue a deeper understanding of the biological and chemical processes which have made the measurement of oxytocin and its functions such a humbling enterprise. 


\section{References}

Alshammari, T. M., Al-Hassan, A. A., Hadda, T. B., \& Aljofan, M. (2015). Comparison of different serum sample extraction methods and their suitability for mass spectrometry analysis. Saudi Pharmaceutical Journal, 23(6), 689-697.

Andreasson, U., Perret-Liaudet, A., van Waalwijk van Doorn, L. J., Blennow, K., Chiasserini, D., Engelborghs, S., et al. (2015). A practical guide to immunoassay method validation. Frontiers in Neurology, 6, 179.

Avanti, C., Hinrichs, W. L., Casini, A., Eissens, A. C., Van Dam, A., Kedrov, A., et al. (2013). The formation of oxytocin dimers is suppressed by the zinc-aspartate-oxytocin complex. Journal of pharmaceutical sciences, 102(6), 1734-1741.

Avanti, C., Permentier, H. P., Dam, A. v., Poole, R., Jiskoot, W., Frijlink, H. W., et al. (2012). A new strategy to stabilize oxytocin in aqueous solutions: li. Suppression of cysteinemediated intermolecular reactions by a combination of divalent metal ions and citrate. Molecular pharmaceutics, 9(3), 554-562.

Bienboire-Frosini, C., Chabaud, C., Cozzi, A., Codecasa, E., \& Pageat, P. (2017). Validation of a commercially available enzyme immunoassay for the determination of oxytocin in plasma samples from seven domestic animal species. Frontiers in Neuroscience, 11, 524.

Blackburn, R. E., Demko, A., Hoffman, G. E., Stricker, E. M., \& Verbalis, J. G. (1992). Central oxytocin inhibition of angiotensin-induced salt appetite in rats. American Journal of Physiology-Regulatory, Integrative and Comparative Physiology, 263(6), R1347-R1353.

Brandtzaeg, O. K., Johnsen, E., Roberg-Larsen, H., Seip, K. F., MacLean, E. L., Gesquiere, L. R., et al. (2016). Proteomics tools reveal startlingly high amounts of oxytocin in plasma and serum. Scientific Reports, 6, 31693.

Caldwell, J. D., \& Jirikowski, G. F. (2014). Sex hormone binding globulin and corticosteroid binding globulin as major effectors of steroid action. Steroids, 81, 13-16.

Caldwell, J. D., Suleman, F., Chou, S.-H., Shapiro, R., Herbert, Z., \& Jirikowski, G. (2006). Emerging roles of steroid-binding globulins. Hormone and metabolic research, 38(04), 206-218.

Carter, C., Pournajafi-Nazarloo, P., Kramer, K. M., Ziegler, T. E., White-Traut, R., Bello, D., et al. (2007). Oxytocin: Behavioral associations and potential as a salivary biomarker. New York: New York Academy of Sciences, 1098(1), 312-322.

Carter, C. S. (2014). Oxytocin pathways and the evolution of human behavior. Annual Review of Psychology, 65, 17-39.

Carter, C. S. (2017). The oxytocin-vasopressin pathway in the context of love and fear. Frontiers in Endocrinology, 8, 356.

Carter, C. S., \& Perkeybile, A. M. (2018). The monogamy paradox: What do love and sex have to do with it? Frontiers in Ecology and Evolution, 6, 202. 
Chard, T., Boyd, N. R., Forsling, M., McNeilly, A., \& Landon, J. (1970). The development of a radioimmunoassay for oxytocin: The extraction of oxytocin from plasma, and its measurement during parturition in human and goat blood. Journal of Endocrinology, 48(2), 223-234.

Dawood, M. Y., Raghavan, K., Pociask, C., \& Fuchs, F. (1978). Oxytocin in human pregnancy and parturition. Obstetrics \& Gynecology, 51(2), 138-143.

de Jong, T. R., Menon, R., Bludau, A., Grund, T., Biermeier, V., Klampfl, S. M., et al. (2015). Salivary oxytocin concentrations in response to running, sexual self-stimulation, breastfeeding and the tsst: The Regensburg oxytocin challenge (ROC) study. Psychoneuroendocrinology, 62, 381-388.

du Vigneaud, V. (1956). Trail of sulfur research: From insulin to oxytocin. Science, 123(3205), 967-974.

Feldman, R., Weller, A., Zagoory-Sharon, O., \& Levine, A. (2007). Evidence for a neuroendocrinological foundation of human affiliation plasma oxytocin levels across pregnancy and the postpartum period predict mother-infant bonding. Psychological Science, 18(11), 965-970.

Franke, A. A., Li, X., Menden, A., Lee, M. R., \& Lai, J. F. (2019). Oxytocin analysis from human serum, urine, and saliva by orbitrap liquid chromatography-mass spectrometry. Drug testing and analysis, 11(1), 119-128.

Galeandro, L., Sieber-Ruckstuhl, N., Riond, B., Hartnack, S., Hofmann-Lehmann, R., Reusch, C., et al. (2014). Urinary corticoid concentrations measured by 5 different immunoassays and gas chromatography-mass spectrometry in healthy dogs and dogs with hypercortisolism at home and in the hospital. Journal of Veterinary Internal Medicine, 28(5), 1433-1441.

Green, L., Fein, D., Modahl, C., Feinstein, C., Waterhouse, L., \& Morris, M. (2001). Oxytocin and autistic disorder: Alterations in peptide forms. Biological Psychiatry, 50(8), 609-613.

Grinevich, V., Knobloch-Bollmann, H. S., Eliava, M., Busnelli, M., \& Chini, B. (2016). Assembling the puzzle: Pathways of oxytocin signaling in the brain. Biological Psychiatry, 79(3), 155-164.

Hennion, M.-C. (1999). Solid-phase extraction: Method development, sorbents, and coupling with liquid chromatography. Journal of Chromatography A, 856(1-2), 3-54.

Horvat-Gordon, M., Granger, D. A., Schwartz, E. B., Nelson, V. J., \& Kivlighan, K. T. (2005). Oxytocin is not a valid biomarker when measured in saliva by immunoassay. Physiology \& Behavior, 84(3), 445-448.

Jurek, B., \& Neumann, I. D. (2018). The oxytocin receptor: From intracellular signaling to behavior. Physiological reviews, 98(3), 1805-1908.

Landgraf, R., \& Neumann, I. D. (2004). Vasopressin and oxytocin release within the brain: A dynamic concept of multiple and variable modes of neuropeptide communication. Frontiers in Neuroendocrinology, 25(3), 150-176. 
Lefevre, A., Mottolese, R., Dirheimer, M., Mottolese, C., Duhamel, J.-R., \& Sirigu, A. (2017). A comparison of methods to measure central and peripheral oxytocin concentrations in human and non-human primates. Scientific Reports, 7(1), 17222.

Leng, G., \& Sabatier, N. (2016). Measuring oxytocin and vasopressin: Bioassays, immunoassays and random numbers. Journal of Neuroendocrinology, 28(10).

Li, T., Wang, P., Wang, S. C., \& Wang, Y.-F. (2017). Approaches mediating oxytocin regulation of the immune system. Frontiers in Immunology, 7, 693.

Liu, D., Han, X., Liu, X., Cheng, M., He, M., Rainer, G., et al. (2019). Measurement of ultra-trace level of intact oxytocin in plasma using salle combined with nano-lc-ms. Journal of Pharmaceutical and Biomedical Analysis.

MacKinnon, A. L., Carter, C. S., Feeley, N., Gold, I., Hayton, B., Santhakumaran, S., et al. (2018). Theory of mind as a link between oxytocin and maternal behavior. Psychoneuroendocrinology, 92, 87-94.

MacLean, E. L., Gesquiere, L. R., Gee, N., Levy, K., Martin, W. L., \& Carter, C. S. (2018). Validation of salivary oxytocin and vasopressin as biomarkers in domestic dogs. Journal of Neuroscience Methods, 293(1), 67-76.

MacLean, E. L., Gesquiere, L. R., Gee, N. R., Levy, K., Martin, W. L., \& Carter, C. S. (2017). Effects of affiliative human-animal interaction on dog salivary and plasma oxytocin and vasopressin. Frontiers in Psychology, 8, 1606.

MacLean, E. L., Gesquiere, L. R., Gruen, M. E., Sherman, B. L., Martin, W. L., \& Carter, C. S. (2017). Endogenous oxytocin, vasopressin, and aggression in domestic dogs. Frontiers in Psychology, 8, 1613.

Martin, W., \& Carter, C. S. (2013). Oxytocin and vasopressin are sequestered in plasma. World Congress of Neurohypophyseal Hormones Abstracts.

Matthiesen, A. S., Ransjö-Arvidson, A. B., Nissen, E., \& Uvnäs-Moberg, K. (2001). Postpartum maternal oxytocin release by newborns: Effects of infant hand massage and sucking. Birth, 28(1), 13-19.

McCullough, M. E., Churchland, P. S., \& Mendez, A. J. (2013). Problems with measuring peripheral oxytocin: Can the data on oxytocin and human behavior be trusted? Neuroscience \& Biobehavioral Reviews, 37(8), 1485-1492.

Mei, H., Hsieh, Y., Nardo, C., Xu, X., Wang, S., Ng, K., et al. (2003). Investigation of matrix effects in bioanalytical high-performance liquid chromatography/tandem mass spectrometric assays: Application to drug discovery. Rapid Communications in Mass Spectrometry, 17(1), 97-103.

Morin, V., del Castillo, J. R., Authier, S., Ybarra, N., Otis, C., Gauvin, D., et al. (2008). Evidence for non-linear pharmacokinetics of oxytocin in anesthetizetized rat. Journal of Pharmacy \& Pharmaceutical Sciences, 11(4), 12-24. 
Paquin, J., Danalache, B. A., Jankowski, M., McCann, S. M., \& Gutkowska, J. (2002). Oxytocin induces differentiation of 19 embryonic stem cells to cardiomyocytes. Proceedings of the National Academy of Sciences, 99(14), 9550-9555.

Park, J. Y., \& Kricka, L. J. (2013). Interferences in immunoassay The immunoassay handbook (fourth edition) (pp. 403-416): Elsevier.

Peltola, M. J., Strathearn, L., \& Puura, K. (2018). Oxytocin promotes face-sensitive neural responses to infant and adult faces in mothers. Psychoneuroendocrinology, 91, 261-270.

Rowland, M., Tozer, T. N., Derendorf, H., \& Hochhaus, G. (2011). Clinical pharmacokinetics and pharmacodynamics: Concepts and applications: Wolters Kluwer Health/Lippincott William \& Wilkins Philadelphia, PA.

Rydén, G., \& Sjöholm, I. (1969). Half-life of oxytocin in blood of pregnant and non-pregnant women. Acta Endocrinologica, 61(3), 425-431.

Selby, C. (1999). Interference in immunoassay. Annals of Clinical Biochemistry, 36(6), 704-721.

Stock, S., \& Uvnäs-Moberg, K. (1988). Increased plasma levels of oxytocin in response to afferent electrical stimulation of the sciatic and vagal nerves and in response to touch and pinch in anaesthetized rats. Acta Physiologica, 132(1), 29-34.

Szeto, A., Rossetti, M. A., Mendez, A. J., Noller, C. M., Herderick, E. E., Gonzales, J. A., et al. (2013). Oxytocin administration attenuates atherosclerosis and inflammation in watanabe heritable hyperlipidemic rabbits. Psychoneuroendocrinology, 38(5), 685-693.

Tamma, R., Colaianni, G., Zhu, L.-I., DiBenedetto, A., Greco, G., Montemurro, G., et al. (2009). Oxytocin is an anabolic bone hormone. Proceedings of the National Academy of Sciences, 106(17), 7149-7154.

Tate, J., \& Ward, G. (2004). Interferences in immunoassay. The clinical biochemist reviews, 25(2), 105.

Valstad, M., Alvares, G. A., Egknud, M., Matziorinis, A. M., Andreassen, O. A., Westlye, L. T., et al. (2017). The correlation between central and peripheral oxytocin concentrations: A systematic review and meta-analysis. Neuroscience \& Biobehavioral Reviews, 78, 117124.

Wai, S. T., Siu, A. F., Zhang, Q., \& Chan, H. E. (2018). Maternal oxytocin responsiveness improves specificity of positive social memory recall. Psychoneuroendocrinology.

White-Traut, R., Watanabe, K., Pournajafi-Nazarloo, H., Schwertz, D., Bell, A., \& Carter, C. S. (2009). Detection of salivary oxytocin levels in lactating women. Developmental Psychobiology, 51(4), 367-373.

Wotjak, C., Ganster, J., Kohl, G., Holsboer, F., Landgraf, R., \& Engelmann, M. (1998). Dissociated central and peripheral release of vasopressin, but not oxytocin, in response to repeated swim stress: New insights into the secretory capacities of peptidergic neurons. Neuroscience, 85(4), 1209-1222. 
Yamamoto, Y., Liang, M., Munesue, S., Deguchi, K., Harashima, A., Furuhara, K., et al. (2019). Vascular rage transports oxytocin into the brain to elicit its maternal bonding behaviour in mice. Communications biology, 2(1), 76.

Ziegler, T. E., \& Wittwer, D. J. (2005). Fecal steroid research in the field and laboratory: Improved methods for storage, transport, processing, and analysis. American Journal of Primatology, 67(1), 159-174. 
Table 1. Advantages and disadvantages of different methods of oxytocin quantitation.

\begin{tabular}{|c|l|l|}
\hline Detection Method & \multicolumn{1}{|c|}{ Advantages } & \multicolumn{1}{c|}{ Disadvantages } \\
\hline \multirow{2}{*}{$\begin{array}{c}\text { Immunoassay } \\
\text { (antibody binding) }\end{array}$} & May detect bound analyte & Identity of analyte not confirmed \\
\cline { 2 - 3 } & May detect degraded analyte & Subject to interferences \\
\cline { 2 - 3 } & May detect multiple forms of analyte & Variability between antibodies \\
\hline \multirow{4}{*}{$\begin{array}{c}\text { Mass spectrometry } \\
\text { (mass-to-charge ratio) }\end{array}$} & Highly specific & $\begin{array}{l}\text { May not detect analyte if bound, } \\
\text { degraded, or present in variable } \\
\text { forms }\end{array}$ \\
\cline { 2 - 3 } & High sensitivity & $\begin{array}{l}\text { Matrix effects due to ion } \\
\text { suppression }\end{array}$ \\
\cline { 2 - 3 } & $\begin{array}{l}\text { Simultaneous detection of multiple } \\
\text { analytes }\end{array}$ & $\begin{array}{l}\text { Misidentification of analytes with } \\
\text { similar molecular weights }\end{array}$ \\
\hline
\end{tabular}


Figure 1. Principles of a competitive oxytocin enzyme-linked immunosorbent assay (ELISA). A) A sample with an unknown amount of oxytocin (blue triangles) and a known amount of enzyme-labeled oxytocin (red triangles) are mixed in a well coated with an immobilized oxytocin antibody. B) Oxytocin in the sample and the enzyme-labeled oxytocin compete for binding to the antibody. C) Unbound material is removed from the well. D) A clear substrate is added, which generates a color-producing reaction with the enzyme-labeled oxytocin. The color produced by this reaction is inversely proportional the amount of oxytocin in the sample.

A

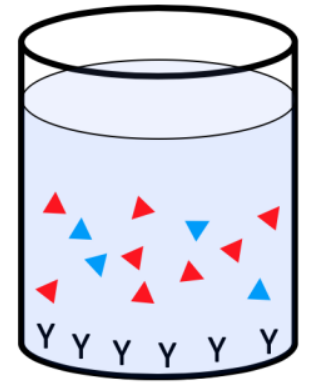

B

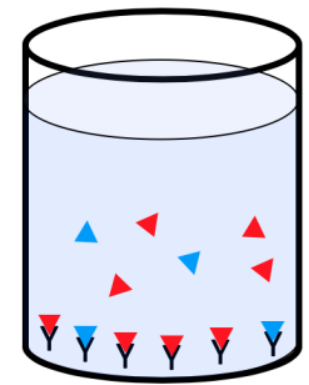

C

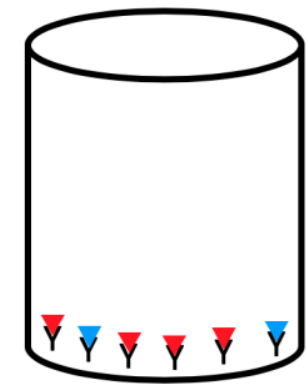

D

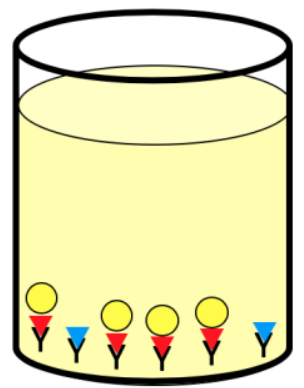


Figure 2. Principles, and challenges of oxytocin extraction. Top: A commonly envisioned ideal scenario of solid phase extraction of oxytocin. A) A biosample (e.g. plasma or serum) including an unknown amount of oxytocin (red triangles), proteins (blue triangles), lipids (yellow circles), and other salts/polar metabolites (green crosses) are introduced to a column filled with particles featuring a hydrophobic surface. B) An aqueous solvent flushes out the salts/polar metabolites, while the oxytocin, proteins and lipids are retained. C) A solvent of intermediate polarity extracts the oxytocin, ready for analysis, while lipids and potentially interfering proteins remain on the column, which is discarded. Bottom: Solid phase extraction of oxytocin where other biomolecules affect the process. A) The same biosample is applied. B) Some oxytocin is bound to proteins, and lipids occupy many of the hydrophobic sites of the column, resulting in free oxytocin being prematurely eluted. C) When oxytocin is finally eluted, only a small fraction is available for analysis.

A

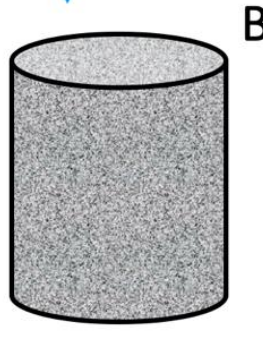

B
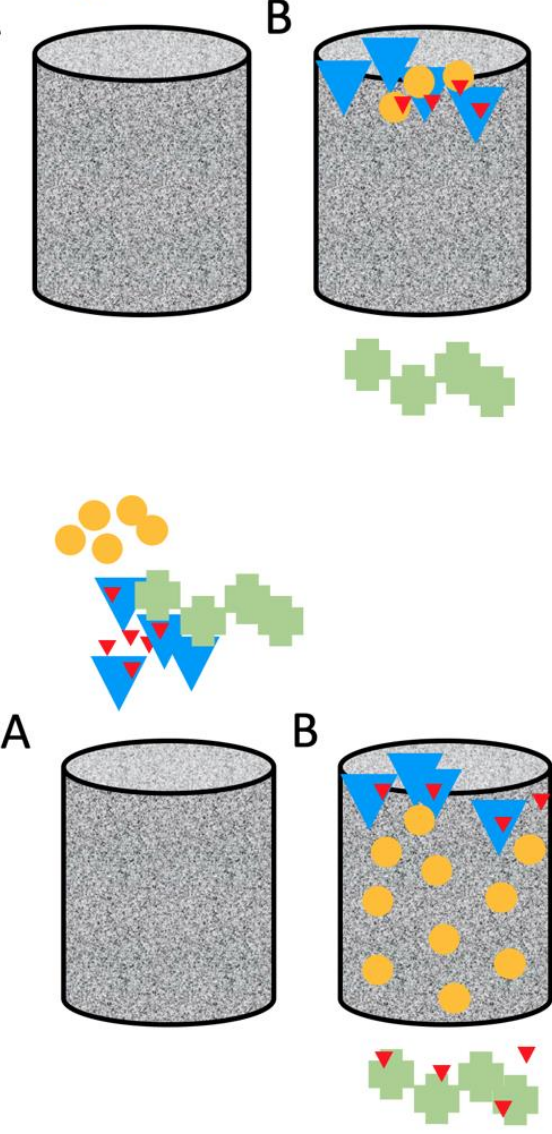

C

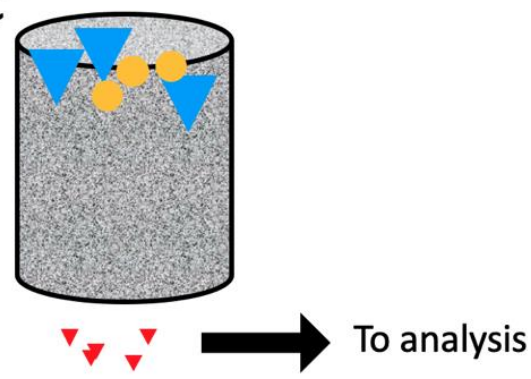

C

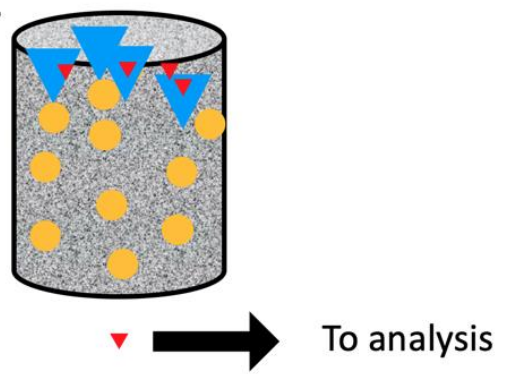


Figure 3. Principles of LC-MS. A) Liquid chromatography: A sample is injected onto a column filled with porous particles, often featuring a hydrophobic surface. The columns are typically around $15 \mathrm{~cm}$ long, with an inner diameter between 0.1 and $2 \mathrm{~mm}$. A solvent, typically including water and methanol, is continuously flushed through the column. Compounds are separated in the column, based on their different affinities to the particles versus the solvent. B) Electrospray ionization (ESI): In LC, compounds are in liquid state, but must be in gas state for subsequent MS analysis. The ESI unit allows fragile biomolecules (such as oxytocin) to enter the gas state as without heating. Briefly, a voltage is applied to the outlet of the fluidic system, allowing LCexiting compounds to be sprayed towards the MS inlet, where they depart from the solvent and into gas phase ions (single or multiple charges). C) Mass spectrometry (MS): The gas state ions enter the MS instrument, where their mass is determined. There are many MS variants, and the figure presents a basic visualization of a Time of Flight (TOF) MS. In TOF, compounds fly through a vacuum tube of a known length. The time of flight then tells us the velocity, and as compounds will have the same kinetic energy in this procedure, the mass is readily calculable. LC-MS provides several key features for identifying of a compound, e.g. the time a compound uses to be chromatographed (the retention time), and its mass. Additionally, serial MS experiments can be performed, e.g. fragmenting the oxytocin mass measured in one MS, and subsequently measuring the fragments' masses with a second MS (tandem MS = MS/MS). MS/MS provides a more comprehensive "picture" of the measured molecule, allowing for more certain analysis. LC-MS (or more precisely, LC-ESI-MS) is standard instrumentation for doping analysis, proteomics, environmental analysis, etc.

\section{A. Liquid chromatography (LC): Separation of compounds}

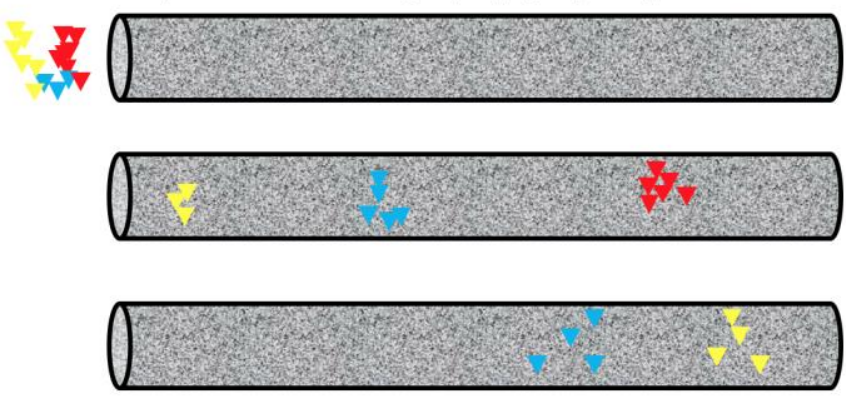

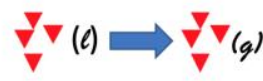
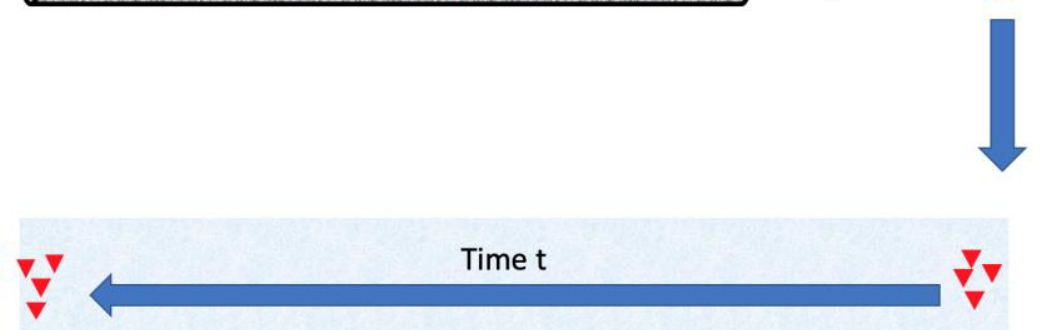

Vaccum tube, length $\mathrm{L}$
B. Electrospray ionization (ESI): Liquid to gas transition

C. Mass spectrometry (MS): "weighing» molecules

$\mathrm{L} / \mathrm{t}=$ velocity

$E_{k}=1 / 2 m v^{2}$ 